\title{
La médecine: un outil à utiliser avec discernement
}

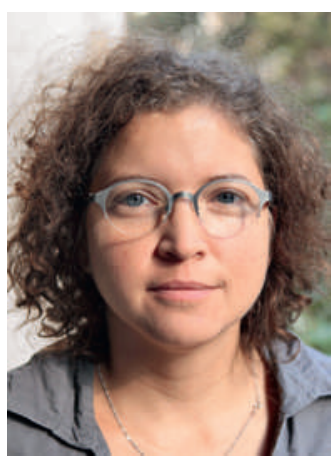

Samia Hurst
Si tout ce que vous avez est un marteau, il est tentant de tout traiter comme des clous. (Abraham Maslow, La psychologie de la science; 1966)

Lorsque l'on parle de «médicalisation», on met en garde contre une tendance à vouloir transformer en problèmes médicaux des situations qui n'en sont pas. Lors de la dernière révision du manuel de diagnostic psychiatrique (DSM 5), on a par exemple assisté à des discussions intenses sur la possibilité de poser un diagnostic de dépression majeure durant une période de deuil, plutôt que de l'exclure comme c'était le cas jusqu'alors [1]. Un des buts était de permettre le remboursement de traitements lorsqu'ils sont bénéfiques pour des personnes dans ces circonstances. Le danger était bien sûr de transformer un aléa douloureux de la vie humaine en problème médical, avec une «solution» médicale.

Bien sûr, les praticiens ne vont pas se mettre juste comme ça à prescrire des antidépresseurs à toutes les personnes endeuillées. Il est cependant important de comprendre à quel point ce souci d'éviter la médicalisation peut être fondé.

Les facteurs qui poussent vers la médicalisation sont nombreux. On répond à la souffrance humaine, avec les moyens à notre disposition. Même lorsque ces moyens ne sont pas adaptés, il reste difficile de ne rien faire. Les intérêts de presque tous convergent vers «plus de médecine». Prescrire davantage, intervenir davantage, c'est souvent gagner davantage d'argent, pour les fabricants vendre plus de médicaments, pour les patients obtenir une réponse humaine à leur souffrance. Nos sociétés ont appris à attendre beaucoup de la médecine. Nous avons en fait même déjà résolu, ou du moins amélioré, de vrais problèmes sociaux. Les épidémies sont devenues rares et sont moins létales. Il est devenu extraordinairement rare de perdre sa mère lors d'un accouchement. On a confiance en la médecine, et on lui en demande davantage. Parfois trop, mais en tout cas les mécanismes de la médicalisation sont durables et rencontrent peu de résistance.

La médicalisation est en général fondée sur des erreurs. Accepter de prendre en charge des problèmes qui ne sont pas médicaux, c'est programmer l'échec. Elle a des effets secondaires: on peut se donner collectivement l'impression de faire quelque chose alors que ce n'est pas le cas. Poursuivre des thérapies sans véritable effet en fin de vie pour continuer de mon- trer au patient qu'on ne l'abandonne pas, ou encore vouloir cibler le burn-out au travail par la seule psychiatrie sans en aborder en même temps le versant social: cela peut être tentant mais ces démarches risquent de devenir des alibis. Comme tous ses instruments, la médecine dans son ensemble est un outil à utiliser avec discernement, uniquement là où elle est appropriée.

\section{«Il reste difficile de ne rien faire.»}

Là où elle est appropriée: nous y voilà. A vouloir éviter de tomber dans la médicalisation des problèmes sociaux, on peut vouloir rejeter en bloc tout ce qui dans la médecine toucherait de près ou de loin à «de la politique». Nous devons cependant avoir conscience que certains usages des outils de la médecine sont légitimes même face à un enjeu de société. Les effets de nos organisations collectives sur la santé, par exemple, sont de mieux en mieux scientifiquement établis, sur plusieurs continents et y compris en Suisse [2-4]. Moins de contrôle sur son travail et ses conditions de vie, cela se traduit en morbidité et en perte d'espérance de vie. Cet effet est désormais sorti du domaine de l'opinion politique pour entrer dans le domaine des faits. On peut diverger sur la manière d'y faire face, de le peser dans nos décisions politiques collectives, mais on ne peut plus le nier. Il nous faut donc aussi être capables de voir quand nos outils sont en fait adaptés: observer, diagnostiquer, signaler, même lorsque l'on ne sait pas comment traiter, voilà des rôles dans lesquels la médecine peut être à sa place.

Samia Hurst*

\section{Références}

1 Living with grief. Lancet. 2012;379(9816):589.

2 Marmot M. The Status Syndrome; How Social Standing Affects Our Health and Longevity. London: Bloomsbury Publishings; 2004.

3 WHO. Socioeconomic determinants of health: the solid facts. Genève: World Health Organization; 2003.

4 Spoerri A, Zwahlen M, Egger M, Gutzwiller F, Minder C, Bopp M. Educational inequalities in life expectancy in German speaking part of Switzerland 1990-1997: Swiss National Cohort. Swiss Med Wkly. 2006; 136(9/10):145-8. 